\title{
Design and construction of a bespoke system for the detection of buried, iron-rich meteorites in Antarctica
}

DOI:

10.1017/S0954102019000531

\section{Document Version}

Accepted author manuscript

Link to publication record in Manchester Research Explorer

\section{Citation for published version (APA):}

Wilson, J. W., Marsh, L., Van Verre, W., Rose, M., Evatt, G., Smedley, A., \& Peyton, A. (2020). Design and construction of a bespoke system for the detection of buried, iron-rich meteorites in Antarctica. Antarctic Science, 32(1), 58-69. https://doi.org/10.1017/S0954102019000531

\section{Published in:}

Antarctic Science

\section{Citing this paper}

Please note that where the full-text provided on Manchester Research Explorer is the Author Accepted Manuscript or Proof version this may differ from the final Published version. If citing, it is advised that you check and use the publisher's definitive version.

\section{General rights}

Copyright and moral rights for the publications made accessible in the Research Explorer are retained by the authors and/or other copyright owners and it is a condition of accessing publications that users recognise and abide by the legal requirements associated with these rights.

\section{Takedown policy}

If you believe that this document breaches copyright please refer to the University of Manchester's Takedown Procedures [http://man.ac.uk/04Y6Bo] or contact uml.scholarlycommunications@manchester.ac.uk providing relevant details, so we can investigate your claim.

\section{OPEN ACCESS}




\section{Design and construction of a bespoke system for}

\section{detection of buried, iron-rich meteorites in Antarctica}

John W Wilson ${ }^{1}$, Liam A Marsh ${ }^{1}$, Wouter van Verre ${ }^{1}$, Michael C Rose ${ }^{3}$, Geoffrey Evatt ${ }^{2}$, Andrew R D Smedley², and Anthony J Peyton ${ }^{1}$

${ }^{1}$ School of Electronic and Electrical Engineering, University of Manchester, M13 9PL, UK

${ }^{2}$ School of Mathematics, University of Manchester, M13 9PL, UK

${ }^{3}$ British Antarctic Survey, High Cross, Cambridge, CB3 0ET, UK 


\begin{abstract}
Iron-rich meteorites are significantly under-represented in collection statistics from Antarctica. This has led to a hypothesis that there is a sparse layer of iron-rich meteorites hidden below the surface of the ice, thereby explaining the apparent shortfall. As standard Antarctic meteorite collecting techniques rely upon a visual surface search approach, the need has thus arisen to develop a system which can detect iron objects under a few tens of centimetres of ice, where the expected number density is of the order one per square kilometre. To help answer this hypothesis, a large scale pulse induction metal detector array has been constructed for deployment in Antarctica. The metal detector array is $6 \mathbf{m}$ wide, able to travel at $15 \mathrm{~km} / \mathrm{h}$ and can scan $1 \mathrm{~km}^{2}$ in around 11 hours. This paper details the construction of the metal detector system with respect to design criteria, notably the ruggedisation of the system for Antarctic deployment. Some preliminary results from UK and Antarctic testing are presented. We show that the system performs as specified, and should reach the pre-agreed target of the detection of a $100 \mathrm{~g}$ iron meteorite at $300 \mathrm{~mm}$ when deployed in Antarctica.
\end{abstract}




\section{Introduction}

Meteorites are found across the globe, with equatorial hot deserts and the cold desert of Antarctica being particularly favourable locations. Despite the inhospitable environment, approximately $65 \%$ of all known meteorites have been retrieved from Antarctica. This is due to the existence of Meteorite Stranding Zones, which are tens of square kilometres sized regions of the ice sheet, which act to concentrate meteorites together upon their blue ice ${ }^{1}$ surfaces (Harvey, 2003).

Broadly, meteorites can be separated into 'iron' and 'stony-iron' (high metallic content or iron-rich) and 'stony' (lower metallic content or iron-poor) classifications. The iron-rich samples account for $5 \%$ of finds in the rest of the world. However in Antarctica iron-rich meteorites occur at a significantly lower proportion, at only $0.5 \%$. This deficit has given rise to the hypothesis of 'lost' iron meteorites within the ice sheet (Evatt et al., 2016).

The previous work by Evatt et al. (2016) explored this deficit by way of a radiative transfer approach. They showed the under-representation could be explained by preferential solar heating of iron meteorites, whose high thermal conductivities and mass allow them to melt faster into the ice than stony meteorites, which are thus more prone to remaining upon the ice surface. Accordingly this suggests that stony meteorites will be preferentially found by systematic searches, whilst a sparse layer of iron meteorites lies hidden below the surface (Evatt et al., n.d.). And it is these 'lost' englacial iron meteorites which this present study hopes to help locate.

The blue ice of the Meteorite Stranding Zones is formed over a period of many decades from the accumulation of snow and subsequent compression. Blue ice (and generally other forms of land-based frozen water such as snow and firn) has a negligible electrical conductivity (Bradley, 1957). By contrast, iron-rich meteorites have a significantly higher electrical conductivity (Ip \& Herbert, 1983). Iron-rich meteorites are also magnetic, thus have a much higher magnetic permeability than the surrounding material. This contrast in electrical conductivity and magnetic permeability allows for the potential to use metal detection technology to sense the discontinuity caused when the detector is in the presence of meteorites.

Metal detectors have been used to detect buried or obscured metallic objects for several decades (Nelson, 2004). They have been applied to a diverse range of applications including humanitarian demining, aviation security, food safety, prospecting, archaeology and treasure hunting (Bell, Barrow, \& Miller, 2001; Craig, 2004; Sweet, 2008; Connor \& Scott, 1998). The physical principles of detector operation have been understood for many years, and the technology is fairly mature. However, in recent years metal detector performance has improved significantly as a result of exploitation of recent advances in electrical engineering; of particular

\footnotetext{
${ }^{1}$ Snow-free regions of glacial ice, whose presence is due to the retardation of the ice flow by underlying mountainous terrain (Bintanja, 1999)
} 
significance is the availability of low noise analogue devices, and the benefits provided through digital signal processing algorithms.

A metal detector exploits Faraday's Law of induction to probe a volume for the presence of highconductivity and/or magnetic materials. Detectors can be implemented using either continuous wave (CW) or pulse induction (PI) configurations (Medek, Nicolics, \& Schrottmayer, 2001). Typically, CW detectors operate in the frequency range of $1 \mathrm{kHz}-100 \mathrm{kHz}$, depending on the conductivity of the material that the detector is searching for. In this mode of operation a transmit coil is used to generate a primary magnetic field as shown in Figure 1. This primary field causes currents to circulate in nearby metallic materials; these circulating currents produce a secondary magnetic field. The signal in a second coil is continuously monitored and can then be used to detect the changes caused as a result of the interaction of the primary and secondary fields. In contrast, PI detectors transmit an impulse-like magnetic field and use the receiver to monitor the decay of the secondary magnetic fields.

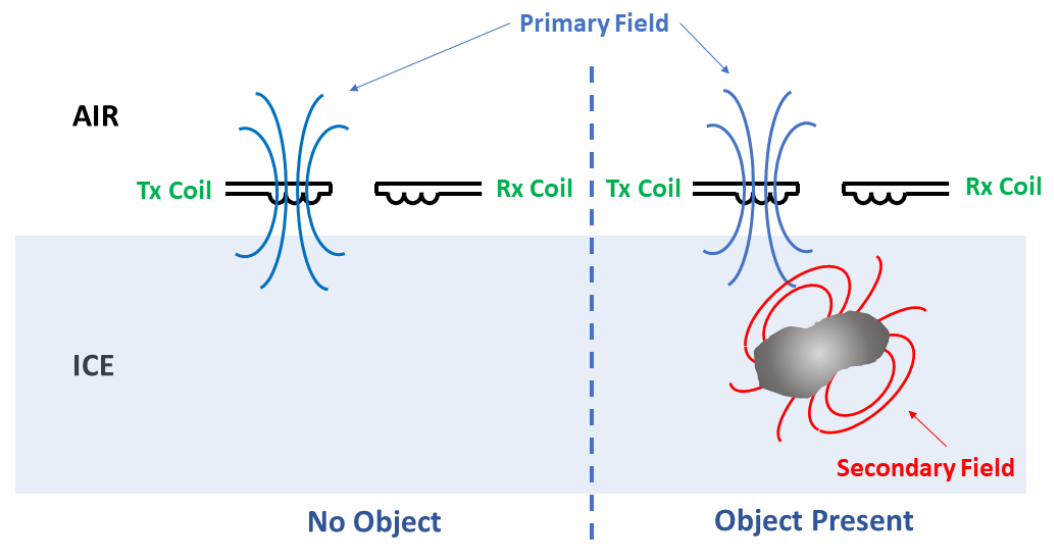

Figure 1: Basic operation of a metal detector

Both methods have advantages and disadvantages. CW detectors offer the highest sensitivity and are typically used for detection on industrial food processing lines; they also offer the potential to resonate excitation / detection signals, thereby taking advantage of natural filtering to reject out of band noise. PI is preferred for applications where sensors are likely to experience vibration during operation. This is because vibration of the sensor causes variations in the primary magnetic field, which in turn causes potential variation in signals coupled into the receive coil. However, pulse methods are able to take advantage of known timedomain profiles of signals to ignore the component of the signal relating to the primary field. This allows the potential for implementing signal processing algorithms which look entirely at the decay in the response of the detector (the decay tail), which is used to indicate the presence of a buried object. Therefore by observing the correct part of the pulse response much of the noise from vibration can be ignored. 


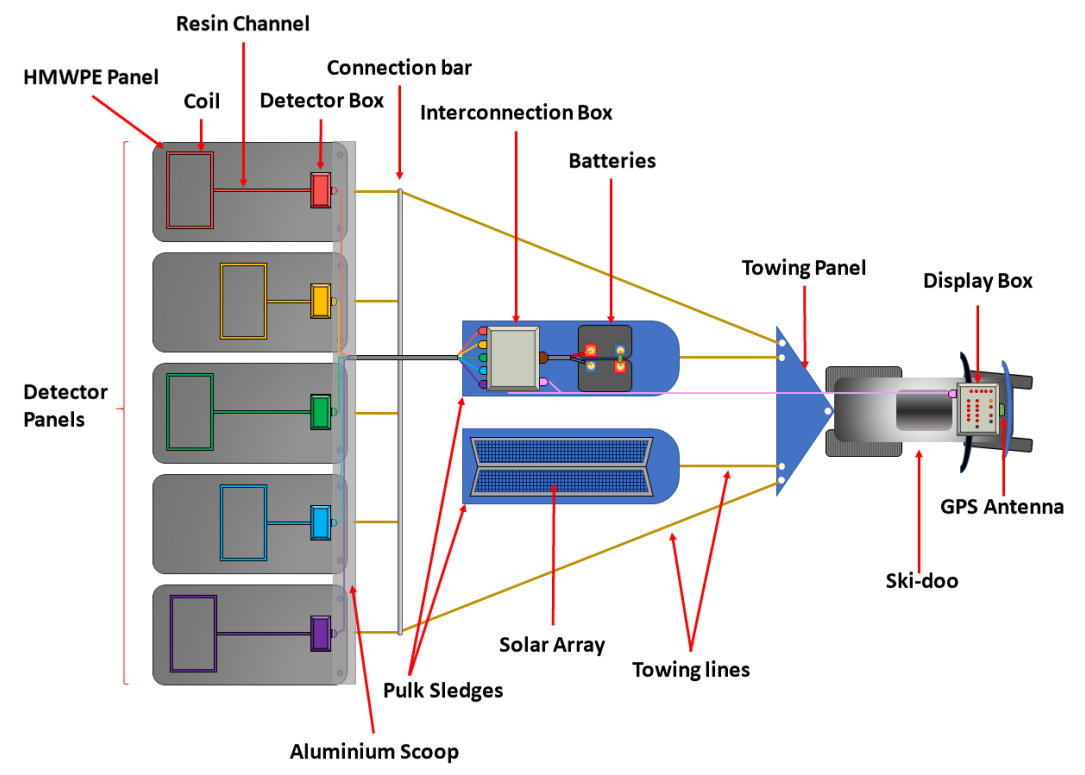

Figure 2: Overview of detector system

The sensor system described in this paper is designed to be towed behind a snowmobile across an icy surface, with the intention of detecting the conductive meteorites buried beneath the surface. Due to the high levels of vibration that are expected in this application PI is a better configuration for the detector system described here.

However after a thorough literature search, and discussions with metal detector manufacturers it was found that no such detection technology was available for use. This has meant the construction of a bespoke meteorite detection system which is both sensitive enough to detect the target objects, and able to withstand the Antarctic environment. This paper describes the design and implementation of such a system.

\section{Overview}

After discussion between the University of Manchester (UoM) and British Antarctic Survey (BAS), the following system specifications were agreed upon. The system should be able to:

- Search a wide area effectively. The expected number density is of the order one iron-rich meteorite per $1 \mathrm{~km}^{2}$ of blue ice (Evatt et al., 2016).

- Be towed behind a snowmobile at a speed of $10-20 \mathrm{~km} / \mathrm{h}$.

- Be disassembled and packed into the cargo space of Twin Otter aircraft.

- Locate an iron rich meteorite, $30 \mathrm{~mm}$ diameter (approximately $100 \mathrm{~g}$ ), trapped within ice at $\leq 300 \mathrm{~mm}$ below the surface. 
- Operate and be stored at temperatures no lower than $-40^{\circ} \mathrm{C}$.

- Withstand high wind speeds (up to $50 \mathrm{~km} / \mathrm{h}$ ) and large amounts of vibration from the scalloped ice surface.

- Operate with real time signal processing with visual and audio alarm output to alert operator and data storage to log position and response.

In order to fulfil the agreed specifications, the configuration shown in Figure 2 was decided upon. The PI metal detector developed at UoM is a modular design featuring five high molecular weight polyethylene panels of the type used to transport fuel in polar regions (Lever \& Weale, 2012). The panels measure $2.0 \mathrm{~m} \times 1.1 \mathrm{~m}$ with a gap of around $0.1 \mathrm{~m}$ between each panel resulting in a detector array with a width of approximately $6 \mathrm{~m}$. This will allow one detector array travelling at an average speed of $15 \mathrm{~km} / \mathrm{h}$ to search approximately $1 \mathrm{~km}^{2}$ in 11 hours.

Each panel contains a coil connected to a self-contained metal detector unit which provides the pulse excitation to the coil, processes the response of the coil to any metal objects within range and transmits a single value derived from the pulse response at a rate of $240 \mathrm{~Hz}$ via an Ethernet connection.

The interconnection box is mounted on one of the two pulk sledges and distributes power from the battery and solar photovoltaic panels mounted on the other pulk sledge to the metal detector units and display unit, transmits a synchronisation pulse to each of the metal detector units, and combines the data streams from each detector unit using an Ethernet switch.

The display box in mounted on the front of the snowmobile within the field of vision and easy reach of the operator. It receives the data streams from all of the metal detector units and when prompted by the operator records them for later analysis. The data is also processed in real time in order to provide a visual and audio cue to the operator when a meteorite is detected by any of the detector units, identifying which panel has passed over the object.

All parts the system were selected with reference to the agreed specifications, rated to operate at $-40^{\circ} \mathrm{C}$ or less down to printed circuit board (PCB) component level. Boxes are IP66 rated with PCBs, sockets, etc. mounted using nylon locking nuts to increase resistance to vibration. Sockets, cables and connectors were selected for their resistance to harsh environments. 


\section{System Design}

\subsection{Coil Simulation}
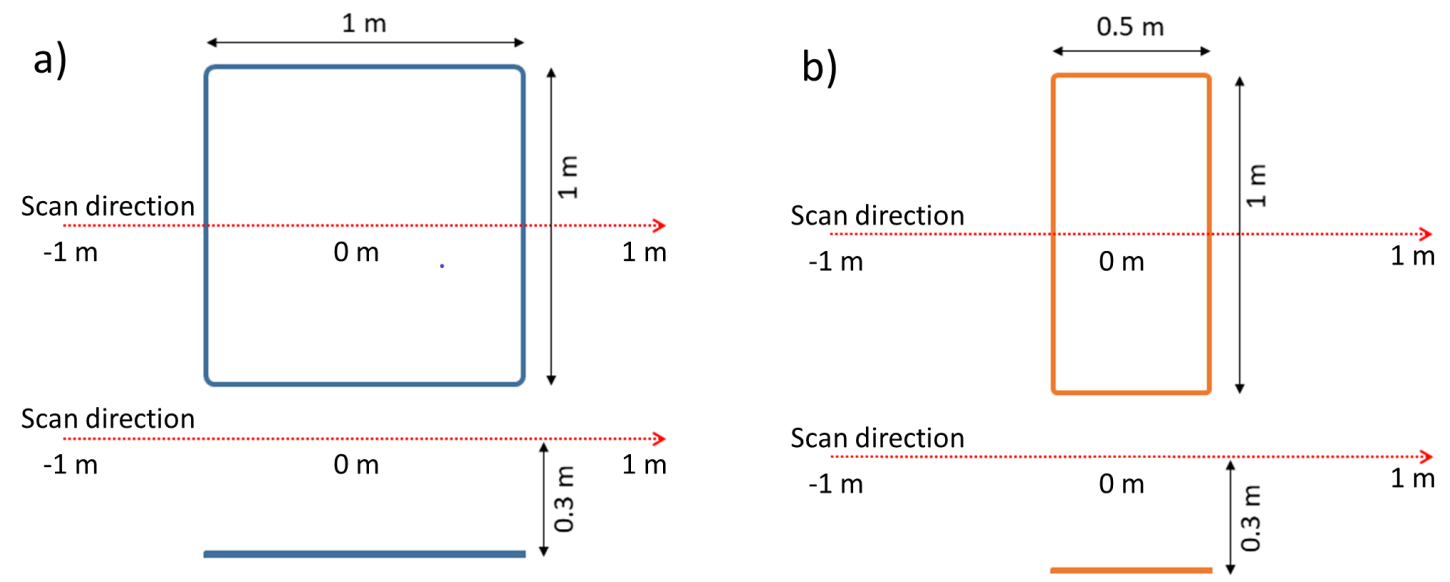

Figure 3: Coil simulation configuration (a) $1 \mathrm{~m} \mathrm{x} 1 \mathrm{~m}$ coil, (b) $1 \mathrm{~m} \times 0.5 \mathrm{~m}$ coil

A number of electromagnetic simulations were undertaken in order to determine the optimum coil configuration. A major constraint is posed by the fixed width of the panels; this is dictated by the limited space on a Twin Otter aircraft, which will be used to deploy the final system in Antarctica. In order to quickly, and efficiently scan a large area, it was determined that the largest possible coil width should be employed. However, there is an important trade-off between the optimum sensitivity depth and coil dimensions. Generally speaking as coil area increases the sensitivity to larger objects also increases (at the expense of sensitivity to smaller objects); the magnetic field produced by the coil will also have a greater penetration depth, thereby allowing the potential to sense deeper and larger targets. It was consequently decided to allow a coil of the maximum permissible width $(1 \mathrm{~m})$, and to tune the overall length according to the optimal sensitivity to the desired target object.

The simulation was used to predict the system response based on the implementation of Biot-Savart law, and using the magnetic polarisability tensor as described in (Marsh, Ktistis, Järvi, Armitage, \& Peyton, 2013) to represent the target object. A spherical target approximation was used to represent the meteorite, and a variety of different coil configurations were examined. Figure 3 shows two of the geometries investigated, namely the $1 \mathrm{~m} \mathrm{x} 1 \mathrm{~m}$, and $1 \mathrm{~m} \times 0.5 \mathrm{~m}$ coils. In each simulation an object is scanned across the centre of the coil with respect to the $1 \mathrm{~m}$ width with the distance between coil and object set at $300 \mathrm{~mm}$. 


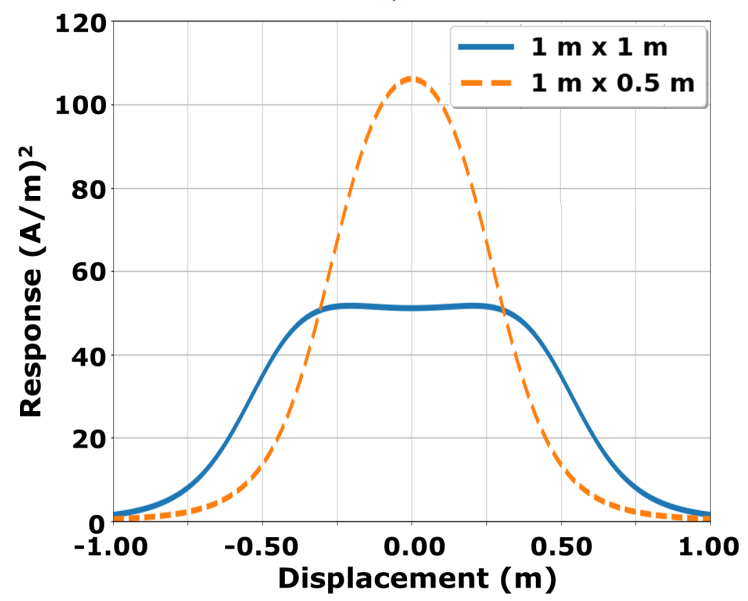

Figure 4: Simulated response to spherical target at a depth of $30 \mathrm{~cm}$

The results of the simulations are shown in Figure 4. It is possible to see that the coil of $1 \mathrm{~m}$ length is able to detect the meteorite for longer, demonstrated by the fact that the response is significantly above zero in the range of $\pm 0.6 \mathrm{~m}$, whereas the coil of $0.5 \mathrm{~m}$ length only demonstrates an equivalent non-zero sensitivity range of $\pm 0.3 \mathrm{~m}$. However, the sensitivity to the meteorite for the $0.5 \mathrm{~m}$ coil is significantly higher than the $1 \mathrm{~m}$ coil when the target is directly beneath the centre of the coil ( $0.00 \mathrm{~m}$ displacement). This is indicative of the fact that a $0.5 \mathrm{~m}$ coil is better suited to detecting objects at the target depth of $300 \mathrm{~mm}$; it is known that the sensitivity as a function of object depth for this coil will tend towards zero more sharply than for the $1 \mathrm{~m}$ equivalent. As the specification for the system requires detection of meteorites down to $300 \mathrm{~mm}$ it is desired to select the coil design which offers the best sensitivity in this range. Consequently the $1 \mathrm{~m} \times 0.5$ $\mathrm{m}$ coil was selected as the optimal design for this depth range.

\subsection{Panel Design}

The design uses a single coil to transmit the excitation pulse and receive the signal from the buried object. The coil is constructed from 15 turns of $0.22 \mathrm{~mm}^{2} \mathrm{PVC}$ insulated copper wire with the coil encapsulated into each panel using two part polyurethane potting compound as shown in Figure 5. As shown in Figure 6, the coils are offset on neighbouring panels to reduce interference between adjacent coils. 


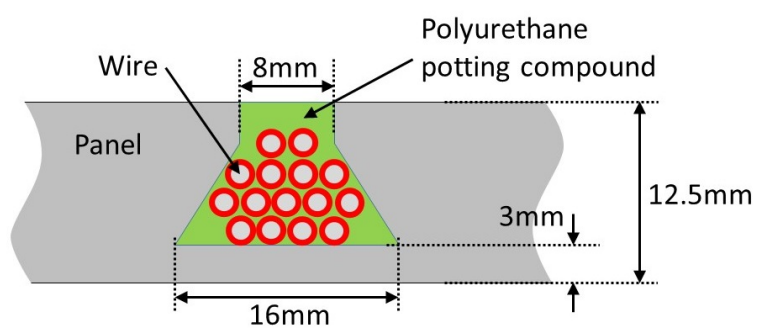

Figure 5: Side view of channels in panel encapsulating coils

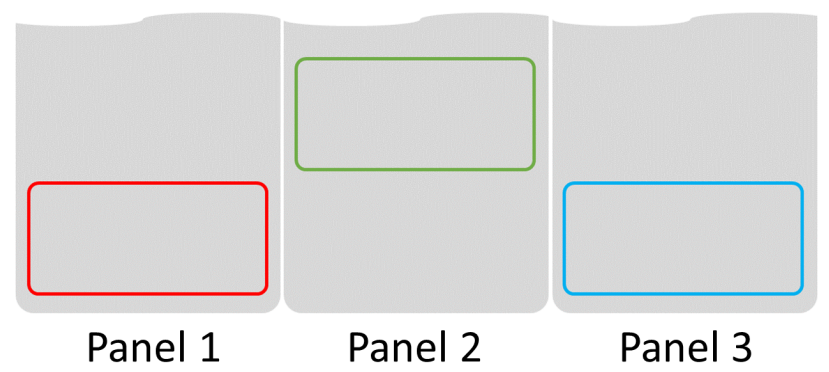

Figure 6: Arrangement for staggering of neighbouring coils to minimise cross-coupling

Each panel also contains a temperature sensor and accelerometer which are used for diagnostic purposes, and to monitor the impact of temperature and vibration on the system. Using this arrangement it is possible to discount any apparent metal detector signals that occur as a result of sharp impacts on the panel, rather than from metallic targets.

\subsection{Metal Detector}

The metal detector unit is controlled by a Red Pitaya single board computer (SBC1). This device was chosen for its $14 \mathrm{bit}, 125 \mathrm{MS} / \mathrm{s}$ analogue-to-digital convertors required for accurate measurement of the response signal and its powerful on-board processor needed to process the detector response for real-time detection. It was temperature tested in-house as fully operational down to $-30^{\circ} \mathrm{C}$ prior to its inclusion in the system.

Figure 7 shows a block diagram of the metal detector unit. A 1 volt excite pulse with a repetition rate of $1 \mathrm{kHz}$ and a $25 \%$ duty cycle is supplied from SBC1 and applied to the pulse driver circuit. The transmit-coilreceive part of the circuit is of a type widely documented elsewhere (?, ?) and is built around a MOSFET switching circuit on the transmit side and diode clipping to reject the high voltage part of the pulse on the receive side.

Initial amplification is provided by variable gain stage 1 . Figure 8 shows the output of the stage with the gain set low to allow the full pulse response signal through. It can be seen that the presence of a metal object 


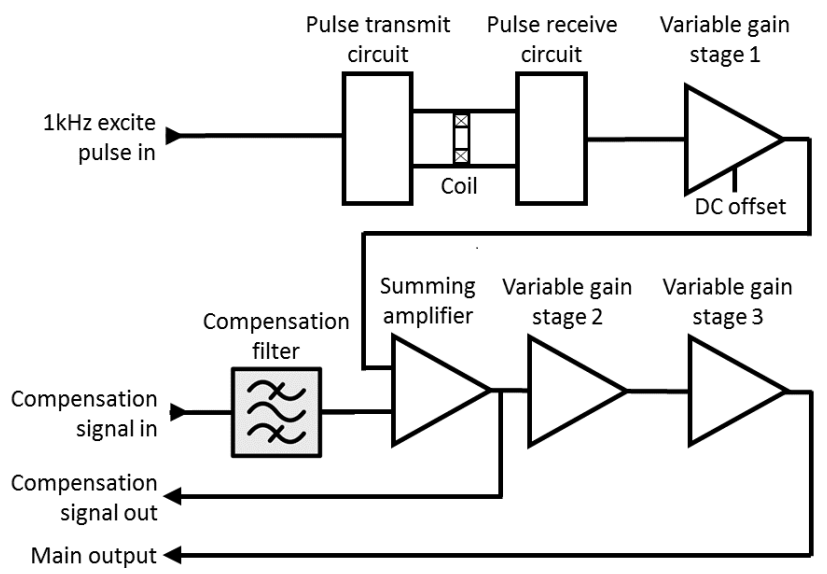

Figure 7: Block diagram of metal detector
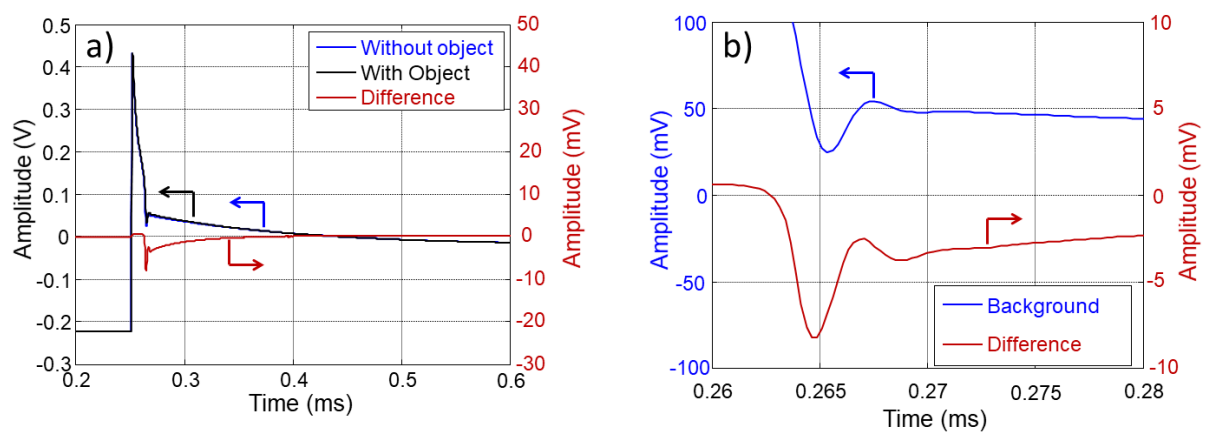

Figure 8: Pulse decay with and without metal object present; a) full scale, b) zoomed

causes a change in the decay of the signal with maximum difference at around $0.265 \mathrm{~ms}$. It is this change in the decay of the signal that is harnessed for metal detection. In normal operation the gain of this stage is set high to amplify the change in the pulse decay. A DC offset is also introduced here in order to zoom in on the part of the signal of interest.

The summing amplifier sums the amplified pulse decay with a compensation signal. The compensation signal is generated in response to the signal received from the output of the summing amplifier and acts to reduce the output of the summing amplifier to near-zero when no object has been detected.

The compensation signal is generated using a proportional controller on SBC1, based on the measured signal after the summing amplifier. The compensation signal is continuously updated such that the voltage at the output of the summing amplifier is near-zero. The speed of convergence can be controlled by changing the feedback gain or by changing rate at which the compensation signal update routine is called, as it is not called for every input signal.

The nulling speed has to be chosen such that it removes changes in the signal due to slow drift phenomena, but at the same the time the nulling loop should not significantly reduce the signal from a buried target. The 
nulling loop also has to limit its response to vibrations in the metal detector system as much as possible.

Two final variable gain stages are included to boost the amplitude of the signal sent back to SBC1 for processing. A band pass filter is applied to the input waveform, primarily to reduce the effect of vibrations. A small number of points are then extracted from the data series and fed through moving-average filters. The detector output is derived from a linear combinations of these points. The exact time indices for these points were derived empirically to maximize object response and minimize the response to vibrations. A combination of band-pass and low-pass filters are used to extract the envelope of the object response in the detector response, and this forms the final output of the system. These filters are highly tuned to the speed of the snowmobile, which should therefore be kept as close to 15 kilometres per hour as possible.

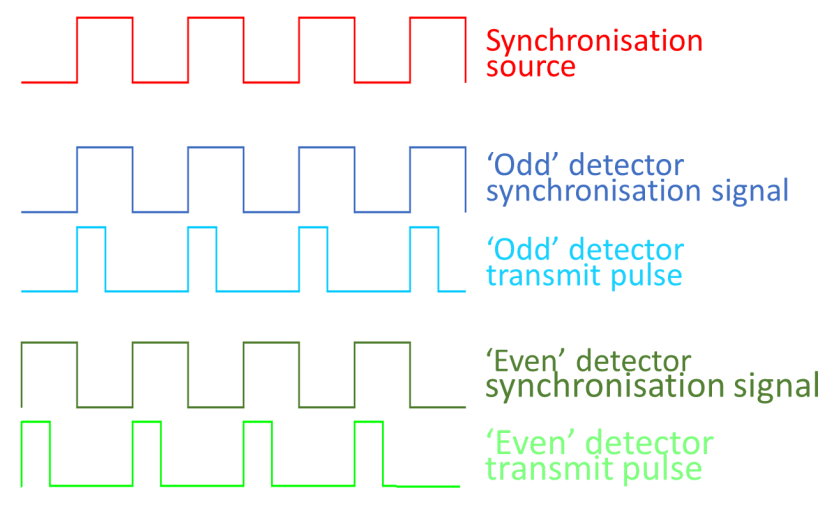

Figure 9: Pulse sequencing to prevent detector cross-talk

In order to further mitigate against detector cross-talk the metal detectors are all synchronised such that neighbouring detectors are not transmitting or receiving simultaneously. Figure 9 shows the sequencing of these pulses. The basic mode of operation is such that a master synchronisation pulse provides a $1 \mathrm{kHz}$ square wave. Several copies of this square wave are buffered, with some being inverted with respect to the master signal. Neighbouring detectors receive different synchronisation signals, with a non-inverted signal being bordered by detectors firing using an inverted signal. Transmit pulses fire for $50 \%$ of the positive half of the detector's synchronisation pulse, and a signal is acquired for the second half of the synchronisation pulse; at all other times the receive signal is ignored. This allows detectors that are not triggered to effectively ignore any induced voltages that originate from neighbouring coils. This, in combination with the aforementioned staggering of coils allows for an effective method of interference-prevention.

\subsection{Display box}

Figure 10 shows the display box for the system. The box is designed to be mounted on the front of the snowmobile within the field of vision and easy reach of the operator. Sealed toggle switches designed for 
outdoor use are employed in the construction along with self-contained LED units rated to IP68. The display box features an external GPS aerial and a sealed $3.5 \mathrm{~mm}$ jack socket for the audio output.

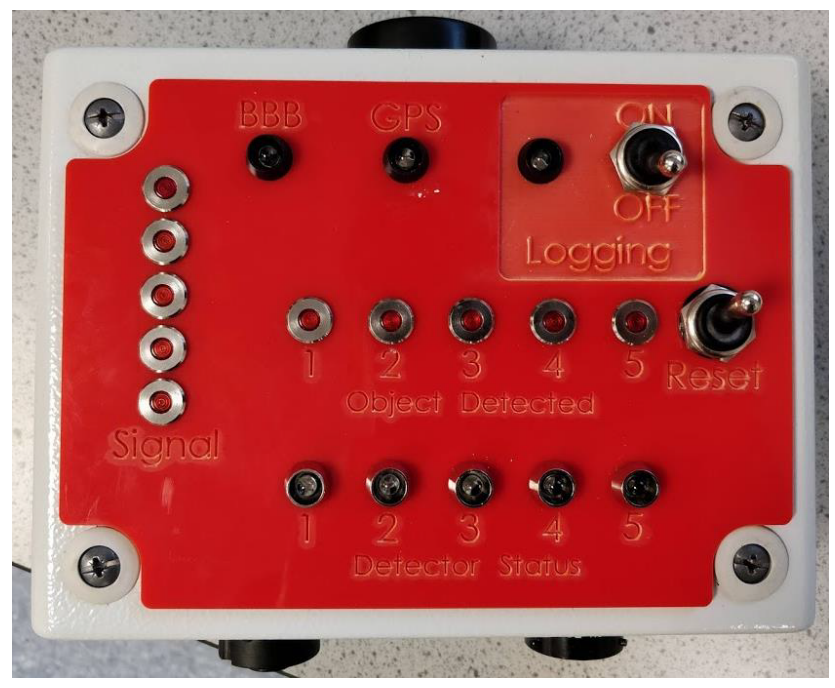

Figure 10: Metal detector display box

The display box contains a BeagleBone Black, a second type of single board computer (SBC2) along with a GPS unit, and some of the signal conditioning hardware required to maintain synchronisation of the metal detector units.

$\mathrm{SBC} 2$ is used as the main interface between the metal detector boxes, and the switches and LEDs mounted on the display box. It also acts as the server for the Ethernet network that is used within the system. Each metal detector box transmits the metal detector response, current box temperature, and vibration data from the accelerometer. This information is stored on $\mathrm{SBC} 2$, along with corresponding GPS data. The LEDs give an indication of the total response of all metal detectors combined, and also provides an indication of which detectors have triggered above a pre-defined threshold; this information is used to aid location of meteorites when the detector is triggered. SBC2 is also responsible for displaying status information such as whether the GPS lock has been acquired, whether the system is currently set to log data, and has some basic user inputs which allow logging to be toggled on and off, and resets any triggered metal detectors. A final key aspect of this box is that it sources the synchronisation pulses which ensure that all metal detector transmit pulses fire in a predefined sequence such as to minimise cross-coupling of signals.

\subsection{Interconnection Box}

The interconnection box provides a central hub for data connections, power distribution and switching. It is located on one of the pulk sledges along with the batteries, with the solar panels located on the other sledge (see Figure 2). It receives the synchronisation pulse from the display box described in section 3.3. This is 
buffered to compensate for the long cable runs and sent out to all the detector units.

The interconnection box also contains an industrial 8-port Ethernet switch to allow the metal detector units to transfer data to the display box and allow the user to interface with any of the metal detector units and the display box.

\section{$4 \quad$ Static Testing}

As a first assessment of the capabilities of the system a number of static tests were carried out using six different iron rich (IAB) meteorites from the Campo del Ciolo meteorite field in Argentina. The samples range in weight from $2.56 \mathrm{~g}$ to $128.1 \mathrm{~g}$. As a guide, the mean composition of Campo del Cielo meteorites is $92.6 \%$ iron, $6.67 \% \mathrm{Ni}$, with remainder being of $\mathrm{Co}, \mathrm{P}, \mathrm{Ga}, \mathrm{Ge}$, and Ir.

As shown in Figure 11, the meteorites were placed in a plastic caddy and pulled across the detector coil on a wooden track with the track placed at different heights ranging from $5 \mathrm{~cm}-60 \mathrm{~cm}$ between the meteorite and the coil. The system used was a simplified version of the system described in Section 3 consisting of a battery, metal detector unit and a laptop for data acquisition.

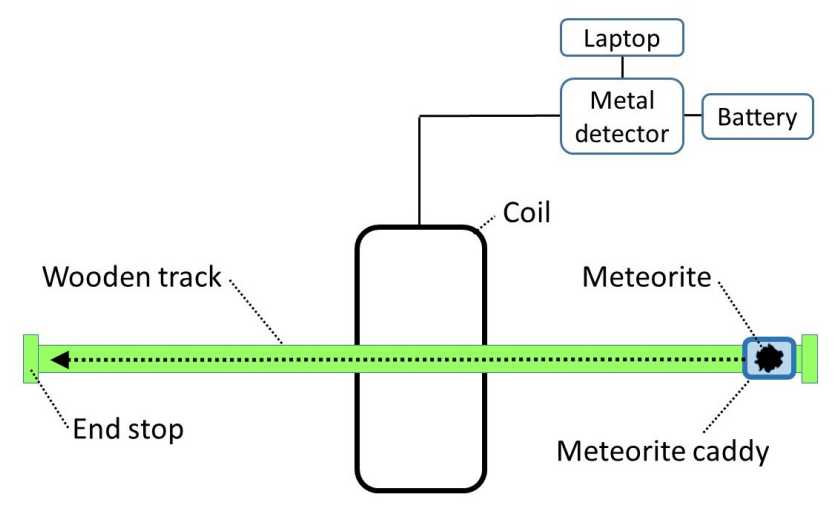

Figure 11: Diagram of top view of static coil meteorite detection tests

Figure 12a shows a plot of the signal amplitude as the $128.1 \mathrm{~g}$ meteorite was passed across the coil at heights from $10 \mathrm{~cm}-60 \mathrm{~cm}$. It can be seen from the plot that at $10 \mathrm{~cm}$ the top of the curve is flattened as the signal through the metal detector circuitry has exceeded that maximum amplitude. The maximum amplitude decreases as distance between coil and object increases reducing to a negligible level at $60 \mathrm{~cm}$.

Figure 12b shows a plot of the peak amplitude of the meteorite response with respect to the distance between coil and object for all six meteorites. It can be seen from the plot that, as would be expected, the response decreases as the the size of the object decreases. The inconsistencies in the response curves can be explained by the irregularly shaped (non-spherical) meteorites invoking different responses when passed through the detector at different orientations with respect to the coil. It can also be seen from the plot that 

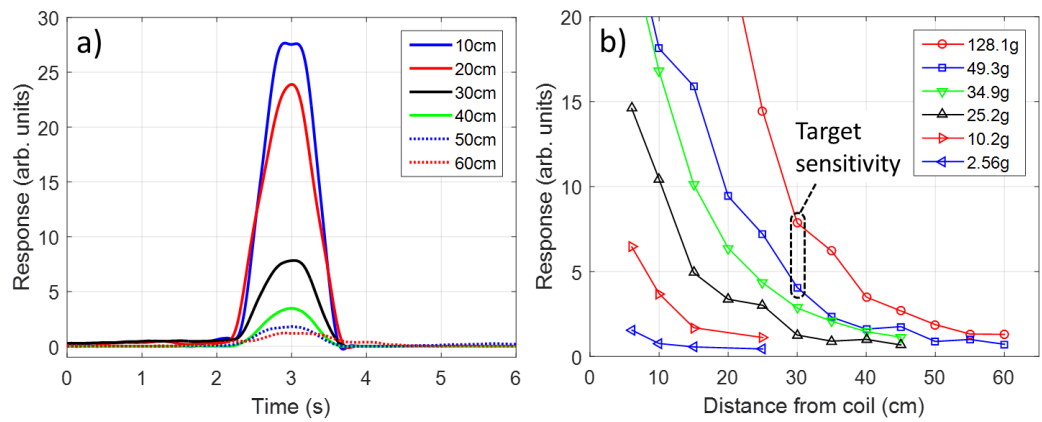

Figure 12: a) Plot of signal response with respect to time as $140 \mathrm{~g}$ meteorite is moved across the coil at different heights, b) Plot of peak amplitude of the meteorite response with respect to height for all six meteorites

the $2.56 \mathrm{~g}$ meteorite invokes some response at $5 \mathrm{~cm}$, although this would fall below the noise floor in the final system whereas $10.2 \mathrm{~g}$ meteorite has a strong response at $\leq 10 \mathrm{~cm}$. At the upper end of the weight range, the $49.3 \mathrm{~g}$ meteorite is clearly detectable at $\leq 30 \mathrm{~cm}$, whereas the response to the $128.1 \mathrm{~g}$ meteorite levels off at distances greater than around $45 \mathrm{~cm}$

The results show that the target of detecting a $100 \mathrm{~g}$ meteorite at a depth of $30 \mathrm{~cm}$ should be attainable using this system. However, it should be noted that this test with a static coil and the meteorite moving with respect to the coil will give a favourable performance for the system. Once vibration and other factors are taken into account performance is expected to decrease significantly.

\section{$5 \quad$ UK Field Testing}

In July 2018 an outdoor trial of the system was conducted in the UK. The test was conducted on an open, grassy surface, and the air temperature was approximately $25^{\circ} \mathrm{C}$; consequently this test was not considered to be fully-representative of conditions in Antarctica. The main purpose of the testing was to provide an evaluation of the performance of the detector when subjected to the stress caused by dragging at representative target speeds. 

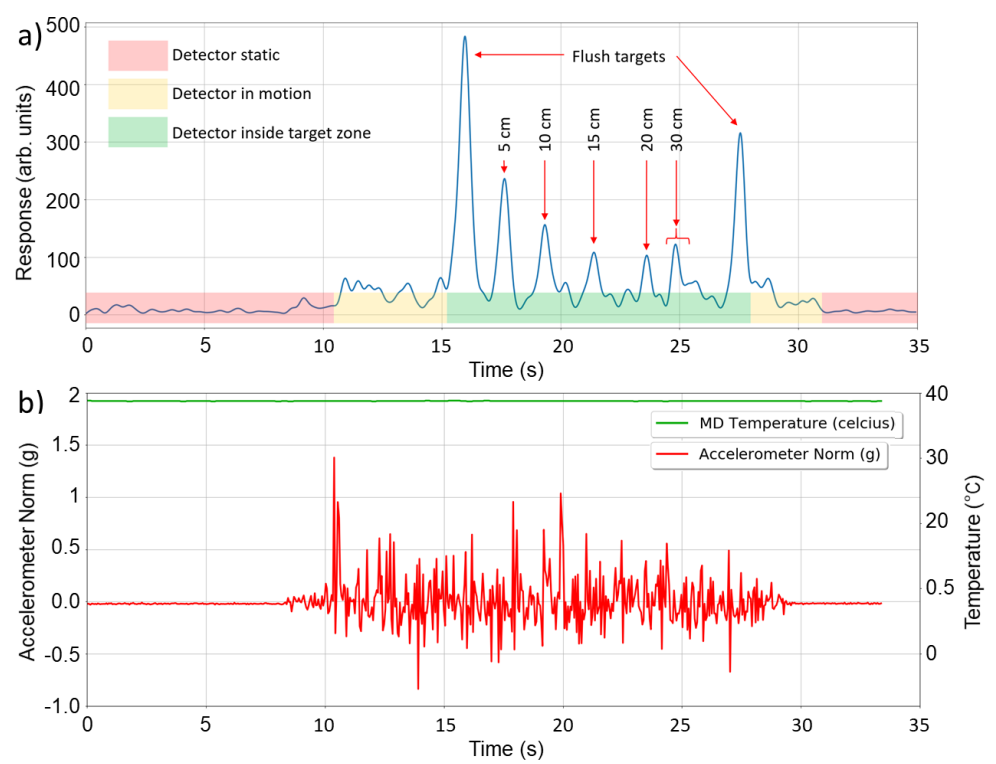

Figure 14: UK outdoor testing: a) Detector results, b) Environmental sensor data

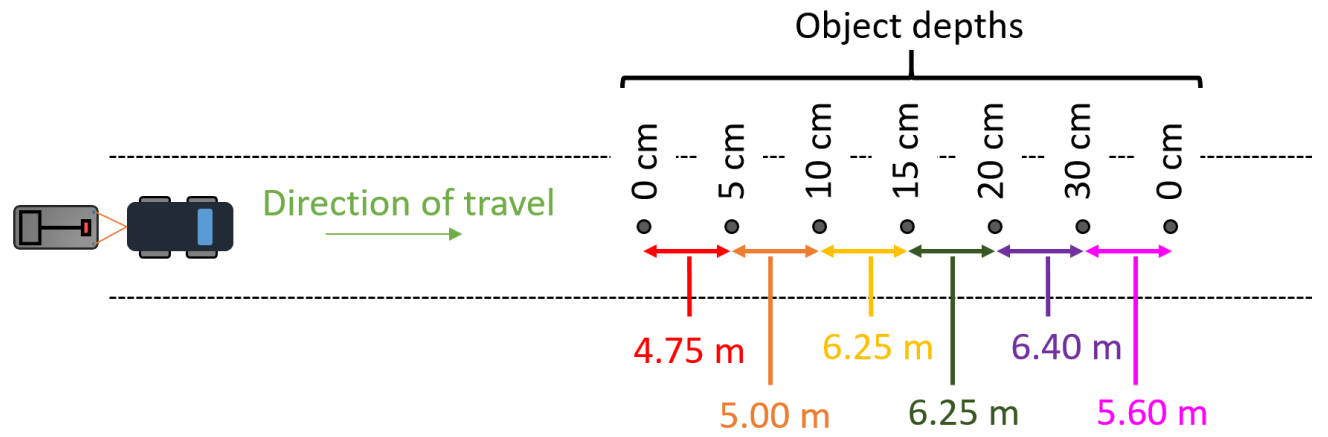

Figure 13: Test Setup - UK outdoor testing

A single coil panel, and metal detector box was connected to the system, and this was towed behind a car; sufficient length was allowed such that the metallic components in the car did not interfere with the performance of the detector. A total of seven $100 \mathrm{~g}$ cylindrical iron meteorite surrogates were buried at depths ranging from $0 \mathrm{~cm}$ (flush to the ground), to $30 \mathrm{~cm}$; the experimental arrangement is shown in Figure 13.

The ground was checked with a handheld metal detector, a Vallon Minehound VMH3CS (Vallon GmbH, n.d.) prior to conducting the test to confirm the mineral content of the ground would not interfere with the detector, and to perform an initial check for any scrap metallic components.

A representative plot showing the detector response is shown in Figure 14a. The regions in which the detector was stationary, moving in a metal-free zone, and moving over the target zones are indicated in shaded red, yellow and green areas respectively. In an ideal case, the response in the red and yellow areas would be indistinguishable from one another; this would demonstrate complete vibration resilience from the 
system. It can be seen that there is a small, yet noticeable difference between these two states. The flush targets, and those buried down to $20 \mathrm{~cm}$ are all clearly visible above the background noise level and follow the anticipated trends. However, the peak in the region labelled as $30 \mathrm{~cm}$ is inconsistent with the expected response. The response would be expected to continue the decreasing trend shown by the other targets, and consequently it should be lower than the peak identified at $20 \mathrm{~cm}$. This peak was measured repeatedly across several scans, an is closer to the $20 \mathrm{~cm}$ peak than would be expected from the experimental setup. The location of the peak does not correlate with any peaks from the accelerometer data, and is therefore not considered to be the result of vibration. The most likely cause for this is that a scrap piece of metallic clutter was present in the ground at this location; given that the signal response is comparable with that of a meteorite surrogate buried to a depth of $20 \mathrm{~cm}$, this could correspond to either a very small object close to the ground, or a larger, deeply-buried object. It is expected that the search with the VMH3CS would find any small metallic targets in the scan region, however as the coil used in the meteorite detection system is considerably larger than the sensor head of the $V M H 3 C S$, it is expected that the meteorite detection system will be more sensitive to objects buried at depth. With this in mind, it is probable that this signal is caused by an object larger than the meteorite surrogate.

The difference in magnitude of the flush targets is not considered to be a cause for concern. A truly identical response would only be observed if the coil took exactly the same trajectory over each target. This, coupled with the fact that the sensitivity gradient is sharpest in regions closest to the coils (thus small positional errors can have a large impact on object response) means that these results are considered valid. The 0 to $20 \mathrm{~cm}$ target responses also demonstrate a typical sensitivity drop-off with respect to distance from the detector.

Figure 14b shows the temperature and accelerometer data for the test shown in Figure 14a. The detector temperature remains at a constant $38^{\circ} \mathrm{C}$. It should be noted that localised heating causes this to be significantly above ambient temperature, and this is something which the final system is designed to cope with during use in polar climates. It is possible to see that the accelerometer measures constant values when the detector is static, however varies significantly during motion, with a peak acceleration of $4.75 \mathrm{~g}$. This peak is caused by the initial motion of the panel as the vehicle sets off. Whilst Figure 14a demonstrated that the detector response was not completely immune to the effects of vibration, it is possible to see that the variation is considerably smaller than that measured by the accelerometer. 

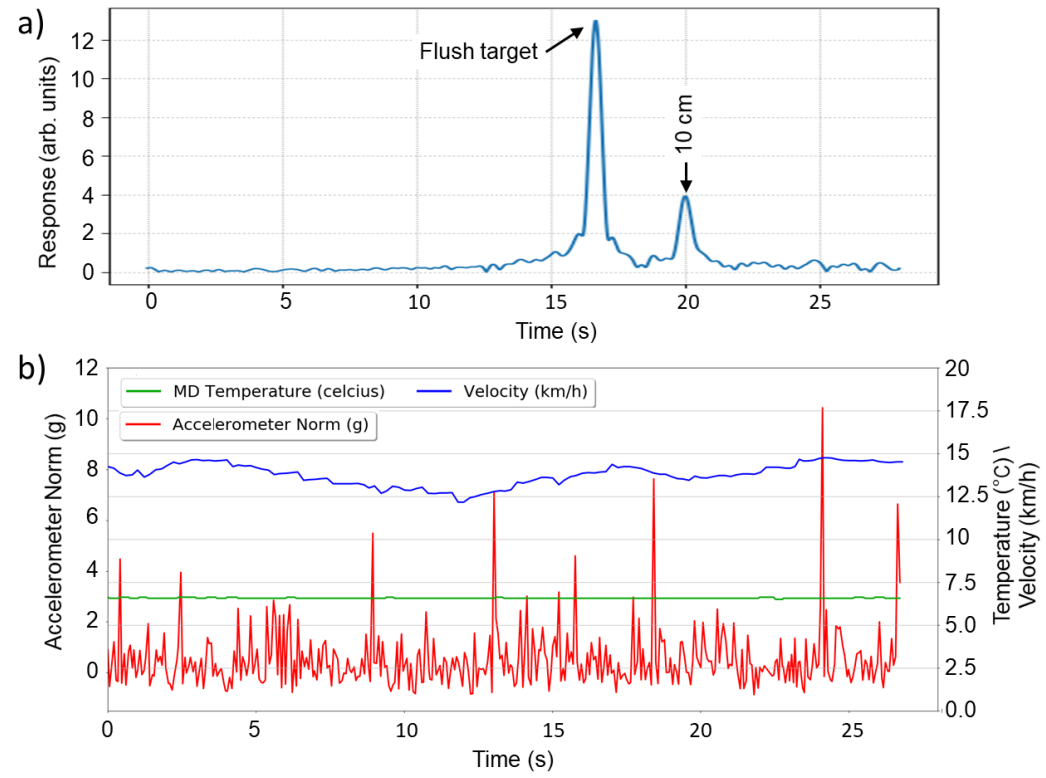

Figure 16: Sky-Blu test: a) Detector results, b) Environmental sensor data

\section{Initial Antarctic Testing}

In January 2019 some preliminary testing of the developed system was carried out at the Sky-Blu field station, Antarctica $\left[74^{\circ} 50^{\prime} 59^{\prime \prime} \mathrm{S} 71^{\circ} 34^{\prime} 0^{\prime \prime} \mathrm{W}\right]$. Figure 15 shows the full five panel metal detector being towed behind a snowmobile, configured as shown in Figure 2.

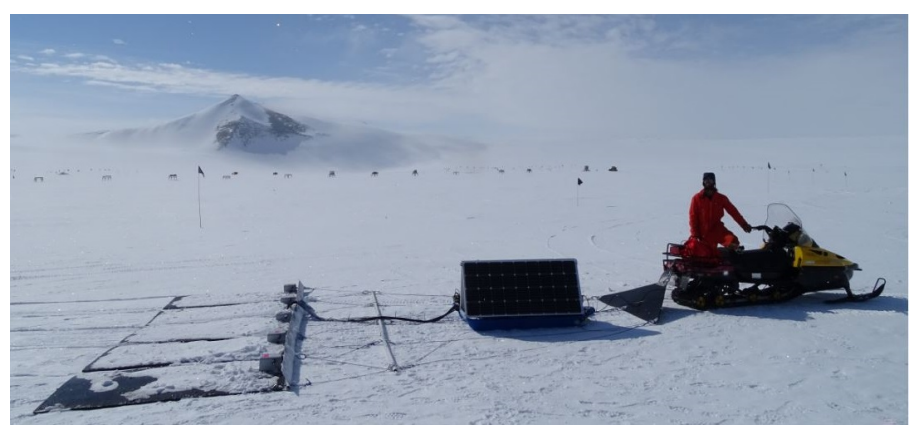

Figure 15: Metal detector tests at Sky-Blu, January 2019

Figure 16a shows some representative data for one of the metal detectors. In this experiment two of the same $100 \mathrm{~g}$ iron surrogates used in the UK-based testing were buried flush to the ice surface, and to a depth of $10 \mathrm{~cm}$. The results show that both targets are clearly detectable above the noise floor of the system. This result is particularly encouraging, as the level of vibration experienced by the detector during these trials was significantly larger than in the UK-based testing; as shown in Figure 16b. This provides confidence in the ability of the system to reject noise due to vibration, as is a fundamental requirement of the final system. 
Full environmental sensor data for the metal detector measurements in Figure 16a is shown in Figure 16b. These results show that the objects remained detectable despite a variation in velocity of approximately $3 \mathrm{~km} / \mathrm{h}$ during the $25 \mathrm{~s}$ scan time. Also of note is the fact that the detector electronics was operating at approximately $7^{\circ} \mathrm{C}$ despite an outdoor air temperature of approximately $-10^{\circ} \mathrm{C}$. It is known that the detectors produce some localised heating, and the electronics has clearly transitioned freezing point to reach this value from a cold start. The fact that the electronics has passed through this condensation point without any concerns is also particularly encouraging.

\section{Summary}

The design and initial testing of a bespoke metal-detection system, to locate iron-rich meteories buried in a subsurface ice layer in the Antarctic, has been detailed in this paper. The system will be used to investigate the underrepresentation of iron-rich meteorities in samples collected from the Antarctic. The future deployment of this system into the Antarctic poses significant challenges and constraints which have to be considered during the design and evaluation process.

For example the requirement that the system should be capable of being disassembled, packed into a Twin Otter aircraft and re-assembled in the field poses constraints on the size of the coils, and the prevailing weather conditions require a wide range of operating and storage temperatures.

The system has been designed with the aim to detect meteorites weighing approximately $100 \mathrm{~g}$ down to $30 \mathrm{~cm}$ below the surface, while the system is moving at a speed of around $15 \mathrm{~km} / \mathrm{h}$. Based on the width of the system $(6 \mathrm{~m})$ and the design speed, it is expected that an area of $1 \mathrm{~km}^{2}$ can be scanned in approximately 11 hours.

Electromagnetic simulations were used to determine the optimal coil size for the detection problem at hand. The width of the coils is constrained at $1 \mathrm{~m}$ due the available space on the Twin Otter aircraft, and it was decided to use the full width available. Based on the simulations it was decided that a coil length of $0.5 \mathrm{~m}$ gives the optimal response to targets buried down to $30 \mathrm{~cm}$.

The metal-detectors are based on PI technology to reduce the impact of vibration-induced noise on the measurements. A summing amplifier is used to back off the background signal, caused mostly by the primary field, electronically using an out-of-phase output calculated and generated by a Red Pitaya single board computer. A polyurethane compound is used to encapsulate the coils into high molecular weight polyethelyne panels measuring $2.0 \mathrm{~m}$ by $1.1 \mathrm{~m}$. The electronics for the PI detector are mounted in a box on the panels, making each panel a self-contained metal-detector. The final system is made up of five panels, with an interconnection box to connect all the panels together. Finally an indicator box is mounted on the 
snowmobile to provide real-time audio-visual feedback to the operator.

Initial tests were performed with the object moving relative to the coil, which was static. This represents the ideal scenario for this system, because the coil is not experiencing any vibration. These tests showed that it is possible to detect $100 \mathrm{~g}$ targets to distances of $30 \mathrm{~cm}$. Subsequent testing was performed in the UK and at Sky-Blu in the Antarctic.

In the UK, a car was used to drag a single panel over a grassy surface with buried objects ranging from $0 \mathrm{~cm}$ to $30 \mathrm{~cm}$ in depth. This test was not a fully representative test, but was used to verify the operation of the system when subjected to the mechanical stresses of being dragged at the target speed. In these tests the system was able to detect objects down to at least $20 \mathrm{~cm}$. While a clear peak in the signal can be seen at $30 \mathrm{~cm}$ the response differs from the expectation, suggesting that it may be caused a piece of metallic clutter rather than the buried target.

In the Antarctic the full mechanical arrangement was evaluated, with five panels being dragged behind a snowmobile at approximately $15 \mathrm{~km} / \mathrm{h}$ over blue ice. The air temperature was also representative of what the final system may encounter at $-10^{\circ} \mathrm{C}$. In these tests the system clearly detected a buried at target at $0 \mathrm{~cm}$ and $10 \mathrm{~cm}$ depth respectively. The outcome of this test was very encouraging as it was the first test of the full system in Antartic conditions.

In both of these tests the acceleration experience by the panel was recorded using an accelerometer. Based on these measurements it appears that the system experiences stronger vibrations on ice than on the grass surface. This suggests that more work may have to be done to harden the final system to vibration, either through mechanical means or in the form of post-processing of the recorded data.

Prior to the deployment of the system to Antarctica, more system testing will be performed in the Arctic in March 2019. Data captured during that trial will be used to further refine the system, in particular the processing algorithms to maximise the detectability of buried targets compared to the vibrations. The final deployment of the system, to search for buried iron-rich meteorites, will take place in 2019-2020.

\section{Acknowledgment}

This work was conducted under 'The Lost Meteorites of Antarctica' grant from Leverhulme Trust no.: RPG2016-349. The authors would also like to recognise the contribution from ESPRC project EP/R002177/1 'Reducing the Threat to Public Safety: Improved metallic object characterisation, location and detection'. 


\section{References}

Bell, T. H., Barrow, B. J., \& Miller, J. T. (2001, June). Subsurface discrimination using electromagnetic induction sensors. IEEE Transactions on Geoscience and Remote Sensing, 39(6), 1286-1293. doi: $10.1109 / 36.927451$

Bintanja, R. (1999). On the glaciological, meteorological, and climatological significance of antarctic blue ice areas. Reviews of Geophysics, 37(3), 337-359. doi: 10.1029/1999RG900007

Bradley, R. S. (1957). The electrical conductivity of ice. Trans. Faraday Soc., 53, 687-691. doi: 10.1039/ TF9575300687

Connor, M., \& Scott, D. D. (1998, Dec). Metal detector use in archaeology: An introduction. Historical Archaeology, 32(4), 76-85. Retrieved from https://doi.org/10.1007/BF03374273 doi: 10.1007/ BF03374273

Craig, J. (2004). 4 - metal detection. In M. Edwards (Ed.), Detecting foreign bodies in food (p. 47 - 62). Woodhead Publishing.

Evatt, G., Coughlan, M., Joy, K., Smedley, A., Connolly, P., \& Abrahams, I. (2016). A potential hidden layer of meteorites below the ice surface of antarctica. Nature communications, 7, 10679. doi: 10.1038/ ncomms10679

Evatt, G., Smedley, A., Joy, K., Hunter, L., Tey, W., Abrahams, I., \& Gerrish, L. (n.d.). The spatial flux of earth's meteorite falls found via antarctic data. Submitted, in review.

Harvey, R. (2003). The origin and significance of antarctic meteorites. Geochemistry, 63(2), 93 - 147. doi: 10.1078/0009-2819-00031

Ip, W. H., \& Herbert, F. (1983, Feb). On the asteroidal conductivities as inferred from meteorites. The moon and the planets, 28(1), 43-47. doi: 10.1007/BF01371671

Lever, J. H., \& Weale, J. C. (2012). High efficiency fuel sleds for polar traverses. Journal of Terramechanics, 49(3-4), 207-213. doi: 10.1016/j.jterra.2012.05.001

Marsh, L. A., Ktistis, C., Järvi, A., Armitage, D. W., \& Peyton, A. J. (2013). Three-dimensional object location and inversion of the magnetic polarizability tensor at a single frequency using a walk-through metal detector. Measurement Science and Technology, 24(4), 045102. doi: 10.1088/0957-0233/24/4/ 045102

Medek, R., Nicolics, J., \& Schrottmayer, D. (2001, May). High sensitive pulse inductive eddy current measurement for mine detection systems. In 24th international spring seminar on electronics technology. concurrent engineering in electronic packaging. isse 2001. conference proceedings (cat. no.01ex492) (p. 207-211). doi: 10.1109/ISSE.2001.931058

Nelson, C. V. (2004). Metal detection and classification technologies. Johns Hopkins APL technical digest, $25(1), 62-67$.

Sweet, K. (2008). Aviation and airport security: Terrorism and safety concerns, second edition. CRC Press.

Vallon GmbH. (n.d.). VMH3CS Mine Detector. http://www.vallon.de/pdf/VMH3CS_Leaflet_EN_2018-05 .pdf/. 